IMTC 2006 - Instrumentation and Measurement

Technology Conference

Sorrento, Italy 24-27 April 2006

\title{
Wireless Sensing for Active Noise Control
}

\author{
L. Sujbert, K. Molnár, Gy. Orosz, and L. Lajkó \\ Department of Measurement and Information Systems, Budapest University of Technology and Economics, and \\ Embedded Information Technology Research Group, Hungarian Academy of Sciences \\ Magyar tudósok krt. 2., 1521 Budapest, Hungary \\ Phone: +36 1 463-2057, Fax: +36 1 463-4112, E-mail: sujbert@mit.bme.hu
}

\begin{abstract}
Our paper introduces a closed-loop wireless control application based on Berkeley MICAz motes and an Analog Devices AD21061 EZ-KIT LITE board. The system realizes active noise control (ANC) for the suppression of periodic acoustic disturbances. The paper highlights the role of synchronous sampling in wireless ANC applications and introduces the solutions in detail. A PLL-like mechanism takes care of the synchronization, while linear interpolation fits the wireless measurement data for processing in the signal processor. The noise controller is a resonator-based one which has proven its efficiency previously in different physical environments. The working of the system is illustrated by measurement results.
\end{abstract}

Keywords - active noise control, wireless control, synchronization, interpolation, resonator-based controller

\section{INTRODUCTION}

Active noise control (ANC) is a known technology for suppression of low-frequency acoustic disturbances which is based on the phenomenon of the destructive interference. A "secondary" noise has to be generated, which suppresses the "primary" (i.e. the original) noise at the properly situated microphones. Although the idea has been known from the beginning of the last century, it became possible to realize only by the spreading of digital signal processors (DSPs). The experiences acquired in the last two decades clarified the possible application area of the principle.

Nowadays wireless network technology over-steps the telecommunication field and appears in instrumentation and control, as well. Active noise control systems comprise many microphones and loudspeakers connected to a controller by wires. Hence, it is a straightforward field for application of wireless networks, as wireless sensors (microphones) and actuators (loudspeakers) are able to ease the deployment of the system and open new possibilities for the acoustic design. Wireless error microphones can be installed on places where cabling was impossible or uncomfortable up to now. In addition, mobile microphones ensure much more flexibility.

Although the utilization of wireless networks would have obvious advantages in numerous applications, it is still in experimental phase. Wireless networks usually cannot satisfy the requirements posed by control systems: real-time or particularly on-line data acquisition is a huge problem. The reason is twofold: the physical bandwidth of the wireless connection is not enough in some applications, and the connection is not reliable.

Wireless control systems can be classified into two groups: open-loop and closed-loop applications [1]. The name "openloop control" is contradictory in a sense. In these systems crucial sensors and actuators are connected to the controller by wires, and wireless connection is used only for such sensors or actuators whose data do not constitute an integral part of the main control loop (e.g. temperature sensors at a rotating machine). Open-loop control has great opportunities in mobile maintenance, redundancy, and ad-hoc benchmarking. In closed-loop applications there are wireless connections in the main control loop, as well. Since it is more crucial, closed-loop applications are still in research phase.

Our active noise control system introduces an example for wireless closed-loop control applications. The controller is a DSP-based signal processing board with an audio codec. The loudspeakers are connected to the DSP by wires, while the microphone signals are transmitted to the DSP by a wireless network. For signal processing and control purposes we have used an Analog Devices AD21061 EZ-KIT LITE board [11] and a set of Berkeley MICAz motes [12] for building wireless connections, as it is described in detail in the following sections.

Most of successful active noise control applications aim the suppression of periodic rather than stochastic noise. First of all, in many situations where high-power acoustic noise is generated, the noise is periodic. Such kind of noise is produced by rotating machines working in factories, motor vehicles, propeller aircrafts, and air conditioners. A classical problem is the noise cancelation around electric transformers. On the other hand, suppression of random noise (e.g. wind noise in a car) is a much more complicated problem, and the existing solutions produce modest results compared to those of periodic noise control. A comprehensive review of the topic can be found in [6]. Hence the implemented ANC aims the cancelation of periodic acoustic disturbances.

Section II. introduces the sensor network, and section III. reviews the noise control algorithm. Section IV. deals with signal processing problems posed by the implementation of ANC 


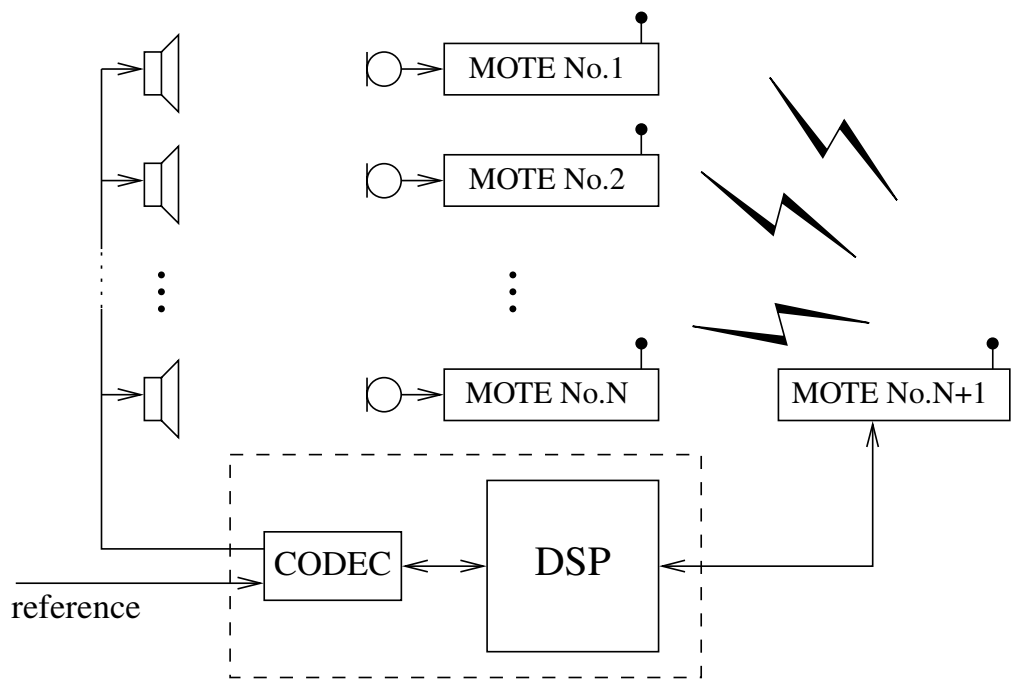

Fig. 1. Block diagram of the system

in a sensor network. Section V. presents the results achieved by the working system.

\section{SYSTEM DESCRIPTION}

\section{A. Hardware components}

The block diagram of our system can be seen in Fig. 1. The controller is an Analog Devices AD21061 EZ-KIT LITE board which integrates the DSP and the codec. The DSP is a floating point processor as it is required for ANC applications. The codec is a 16-bit AD1847 stereo audio codec produced by Ana$\log$ Devices, as well [11]. The loudspeakers are active ones connected by cables to the codec, while the microphones have wireless connection to the DSP. The connection is attained by Berkeley MICAz motes [12] in the following way: N microphones are placed on sensor boards mounted on the MICAz motes, while mote No.N+1 (basestation) is connected to the DSP board by a serial line. The motes send their data packets to the basestation. Most ANC algorithms need a reference signal. The reference input in the figure is optional, it can be also acquired by the motes.

All the motes communicate by ZigBee radio at a carrier frequency of $2.4 \mathrm{GHz}$, and the connection is operational up to $100 \mathrm{~m}$ range [13]. Microphone signals are sampled at $2 \mathrm{kHz}$ frequency and converted by an 8-bit analog to digital converter. This $2 \mathrm{kHz}$ is the global sampling frequency, so ANC is performed up to $1 \mathrm{kHz}$.

\section{B. Topology}

The topology of the sensor network is depicted in Fig. 2. There are three types of messages among the motes. Each mote sends the microphone signal samples to the basestation and it sends all the data to the DSP. The data transfer directions are drawn with a solid line. To avoid message collision, a token-ring topology is built. Whichever mote has the token

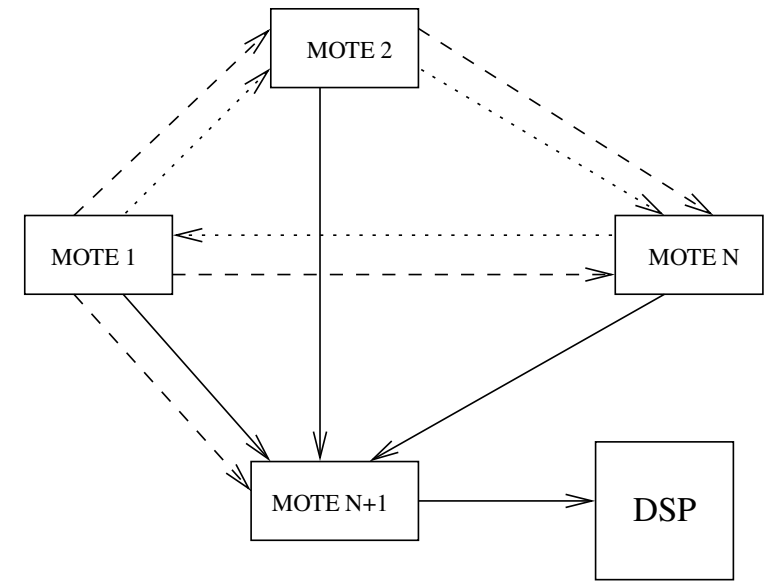

Fig. 2. Network topology. Solid line: data transfer; dotted line: token passing; dashed line: synchronization

can put data into the network. The token passing is denoted with dotted line. An essential function of the network is the synchronization of the motes. The latter only means that the sampling frequency is equal to that of the first mote at each one. The synchronization is discussed in detail in section IV.

The motes send signal samples to the basestation in packets containing 25 samples. There is no acknowledgment after receiving a packet, because an acknowledgment would meaningfully decrease the speed of the transfer. Occasionally missing or bad data are handled on signal processing level.

\section{NOISE CONTROL}

\section{A. Noise control problem}

The basic problem of active noise control can be summarized in a very compact way as it is in Fig. 3. Generally all the signals are vectors, since usually the control system comprises more than one microphone and loudspeaker. The anti-noise 


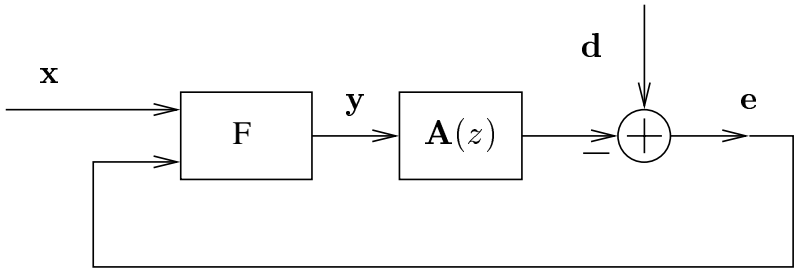

Fig. 3. Noise control problem

is generated by the adaptive system $\mathrm{F}$ (a kind of adaptive filter), the output of which is $\mathbf{y}$. This output signal is radiated by the loudspeakers and subtracted from the noise signal to be suppressed $\mathbf{d}$ at the error microphones. A theoretically important feature of the problem that $\mathbf{y}$ is not directly subtracted but first filtered by $\mathbf{A}(z)$ which is the transfer function matrix between the loudspeakers and the microphones including all ana$\log$ electronic components' transfer functions. $\mathbf{A}(z)$ is usually called secondary path transfer function, since this acoustic path appears just because of the ANC system. The primary path is between the noise source and $\mathbf{d}$. The controller $\mathrm{F}$ in some cases utilizes the reference signal vector $\mathbf{x}$, but its usage is optional. A more detailed introduction and analysis can be found in [6].

There are different approaches for noise controller design. However, all of them need the identification of the secondary path $\mathbf{A}(z)$. Inaccurate model of $\mathbf{A}(z)$ leads to unstable system. The system is stable only if the phase error of the model does not exceed $\pi / 2$ [7], [9]. It is hard condition in certain cases, since only a simple unit delay can cause such an error. If a pure sinusoidal should be suppressed and its frequency is greater than the quarter of the sampling frequency, the phase error exceeds $\pi / 2$ and the control loop is unstable. The latter phenomenon is particularly important in sensor networks and will be considered in section IV.

\section{B. Noise controller}

The ANC implemented in our wireless environment is a resonator-based noise controller. It is designed directly for periodic noise control and has proven to work efficiently [2]. The concept of the controller was successfully applied for a slightly different problem, as well [3]. The key element of the of the structure is the adaptive Fourier analyzer (AFA), which is a structurally adaptive system for exact measurement of band-limited periodic signals of arbitrary fundamental frequency [5]. It is an extension of the resonator based observers developed earlier to perform the recursive discrete Fourier transform (RDFT) [4]. In these observers discrete resonators work in a common feedback loop providing zero steady-state feedback error at the resonator frequencies. The AFA adapts the resonator frequencies to coincide with those in the input signal.

If acoustic noise should be canceled, the output of the resonators should be connected to a loudspeaker and fed back using a microphone. The arrangement can be seen for a one microphone - one loudspeaker system in Fig. 4. The frequency

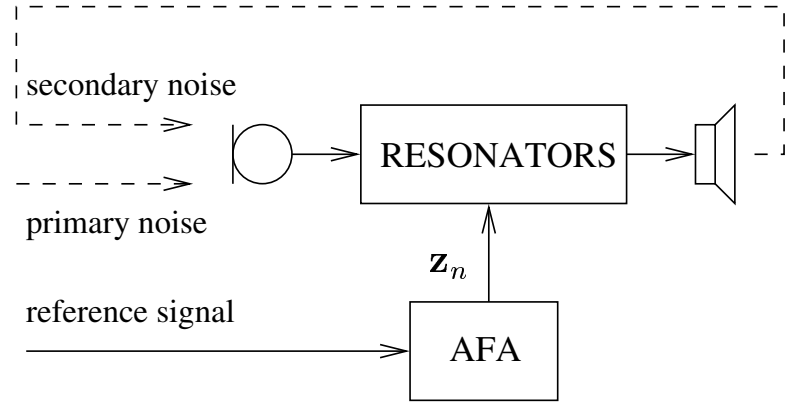

Fig. 4. Periodic noise control: Physical arrangement

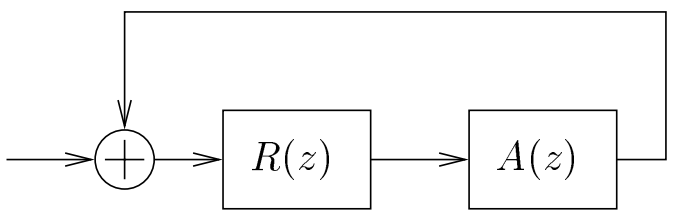

Fig. 5. Periodic noise control: Block diagram

is estimated by an independent AFA and it passes the actual resonator positions $\left(\mathbf{z}_{n}\right)$ to the controller. Reference signal can be any periodic signal with the same fundamental frequency as the primary noise. Fig. 5 shows the block diagram of the control loop, where $R(z)$ and $A(z)$ denote the resonator based controller and the transfer function of the acoustic system, respectively. The stability and fast convergence of the system can be ensured if the resonator channels are multiplied by the following parameters:

$$
w_{k}=\frac{1}{A\left(z_{k}\right)}
$$

where $z_{k}, k=1 \ldots N$ are the resonator frequencies. In the multiple channel system a resonator set belongs to each loudspeaker, and the input of the resonators is the weighted sum of the microphone signals. The weighting of the microphone signals corresponds to the parameters $w_{k}$. Indeed, instead of a simple parameter set, here a matrix set is applied as follows:

$$
\mathbf{W}_{k}=\mathbf{A}^{\#}\left(z_{k}\right)
$$

where $\mathbf{A}(z)$ is the transfer matrix between the loudspeakers and the microphones, $\mathbf{W}_{k}$ is the weighting matrix and \# denotes the pseudo- (or Moore-Penrose) inverse.

\section{SIGNAL PROCESSING}

\section{A. General considerations}

The main objective of signal processing is the implementation of the noise control algorithm. However, by the use of the motes, sampling and data handling is no more obvious.

The motes have poor signal processing capacity: each one has an ATmega128 eight-bit processor running at about 7.4 MHz. On the contrary, the DSP is an ADSP21061 which is a 32-bit floating-point signal processor working at $40 \mathrm{MHz}$. 
Hence the adaptive Fourier analyzer and the noise controller runs on the DSP, and the motes do only the inevitably necessary calculations.

The sampling frequency of the system is controlled by the codec on the DSP board. The codec is connected to a serial port of the DSP. Microphone signal samples arrive to another serial port of the DSP, which the basestation is connected to. The motes are programmed so that their nominal sampling frequency is equal to each other and to that of the DSP board. On the other hand, it is clear that neither the motes nor the DSP have equal sampling frequency in practice, since each mote and the DSP have individual clock generators. Because of the timing error, sampling should be synchronized. The synchronization is achieved in two steps: the sampling at the motes are synchronized to each other, and the DSP interpolates between mote data according to the codec's sampling times.

The necessity of synchronization can be proven in the following way. The motes send data to the basestation and it should send the data to the DSP just after receiving the packet from the slowest mote, otherwise it cannot produce enough new samples. On the other hand, in this case faster motes should store samples not sent to the basestation. This would lead not only to infinite buffers but to the instability of the control system. A faster mote sends those samples which were taken before the sending. The delay between sampling and sending is increasing slowly but infinitely, resulting in an increasing phase error in the model of the secondary path. As the phase error reaches $\pi / 2$ at the highest controlled frequency, the system gets unstable.

\section{B. Synchronization}

Synchronization between the motes is carried out by the receiving of packets in the following way. Mote No.1 is assigned to be the reference mote, data packet of which is received by all the other motes. Sampling at a mote is scheduled by a timer, i.e. realized as a counter. The maximal value of the counter determines the sampling frequency. The value of the counter is a sawtooth signal as a function of time. This is a periodic signal, and its phase is proportional to the actual value of the counter. As the mote receives a data packet from the reference mote, the value of the counter (i.e. the phase of the sawtooth signal) is read. If the sampling frequencies are equal to each other, the same phase is read at each packet arrival. If the mote has greater sampling frequency than that of the reference mote, this phase is increasing and vice versa. Based on this phase information, sampling can be controlled. If the phase is increasing, sampling frequency has to be decreased by writing a greater maximal value into the counter. Decreasing phase calls for opposite action. In other words, synchronization works like a phase locked loop (PLL) as it can be seen in Fig. 6. In the figure $f_{1}$ and $f_{k}$ are the clock frequencies of the first and the $k$ th mote, respectively. $f_{s, 1}$ and $f_{s, k}$ denote the sampling frequencies of the first and the $k$ th mote, respectively. $f_{1} \neq f_{k}$ but in steady state $f_{s, 1}=f_{s, k} . N_{d}$ is the desired value of the counter

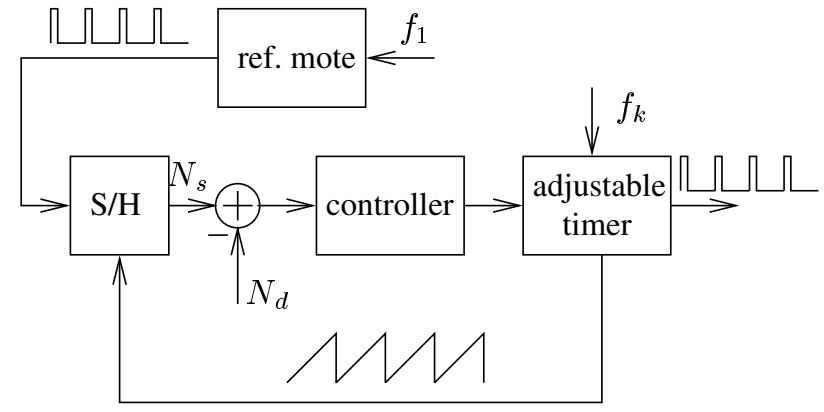

Fig. 6. Synchronization at the motes: $f_{1} \approx f_{k}, f_{s, 1}=f_{s, k}$

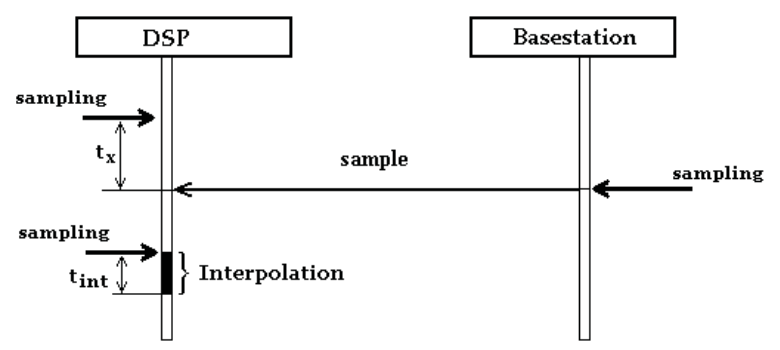

Fig. 7. Interpolation at the DSP

in steady state, while $N_{s}$ is the actual sample of the counter at the $k$ th mote. Each mote is synchronized to the reference mote, so at the end, all the motes have the same sampling frequency as that of the reference mote.

\section{Interpolation}

Since the codec's sampling frequency is not adjustable, the above method of synchronization between the motes and the DSP cannot be utilized. However, the computing capacity of the DSP can be used: the motes' signals are interpolated and re-sampled according to the codec's sampling clock.

The interpolation is carried out in the following way. The DSP measures the time interval $t_{x}$ between the codec and mote data packet arrivals, as it is depicted in Fig. 7. The used interpolation method is simple linear interpolation, as it is satisfactory for our control purposes. Thus the interpolated sample can be calculated in the following way:

$$
y_{n}=x_{n-1}+\frac{t_{x}\left(x_{n}-x_{n-1}\right)}{T_{s}}
$$

where $y_{n}$ is the interpolated value at time instant $n$, while $x_{n}$ and $x_{n-1}$ are the actual and the previous samples, respectively. $T_{s}$ is the sampling interval, the reciprocal of the sampling frequency. Since the Shannon's sampling theorem is satisfied, there is no information loss by this calculation. The procedure is introduced in detail in [10].

At the end, after re-sampling of the signals of the motes, all the signals in the control system are sampled at the time instants determined by the codec. 


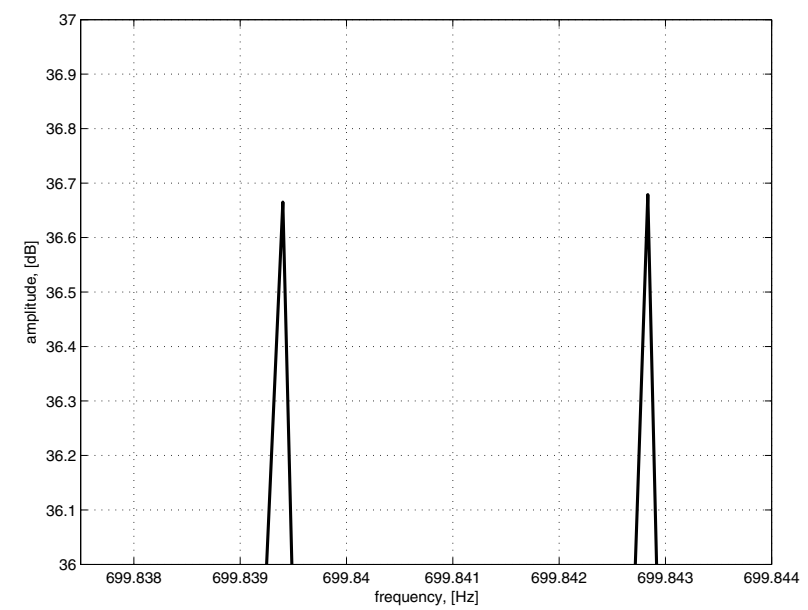

Fig. 8. Spectra of the same sinusoidal waveform received on two motes without synchronization

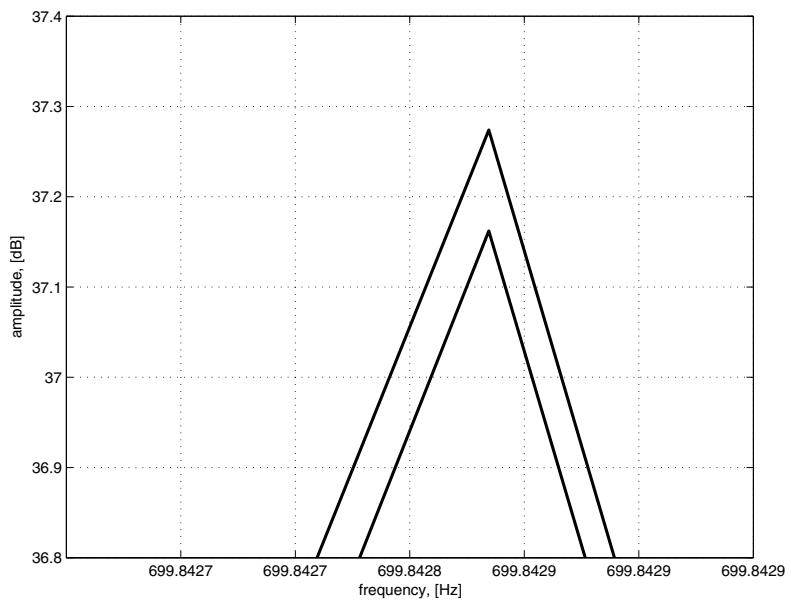

Fig. 9. Spectra of the same sinusoidal waveform received on two motes with synchronization

\section{RESULTS}

Since the synchronization is of key importance, this was tested first. Long records $\left(N=2^{20}\right)$ were sampled with two motes, first without, then with synchronization. The signal was a pure sinusoidal sound received by the motes' microphones. If the sampling frequencies are slightly different, the relative frequencies detected by the motes are also slightly different. Supposing the same nominal sampling frequency, the measured frequencies for the same tone are different. In Fig. 8 this case is illustrated. The two measured frequencies are very close to each other, the relative error is in the range of $10^{-6}$. As stated before, already such a small error can also lead to unstable control. Figure. 9 shows the measurement results in the case of synchronization.

Noise control system was tested in different ways. Both wired and wireless reference signal access was successfully tested. Several acoustic arrangements were prepared in a laboratory room, where many reflections appear. (The experiments

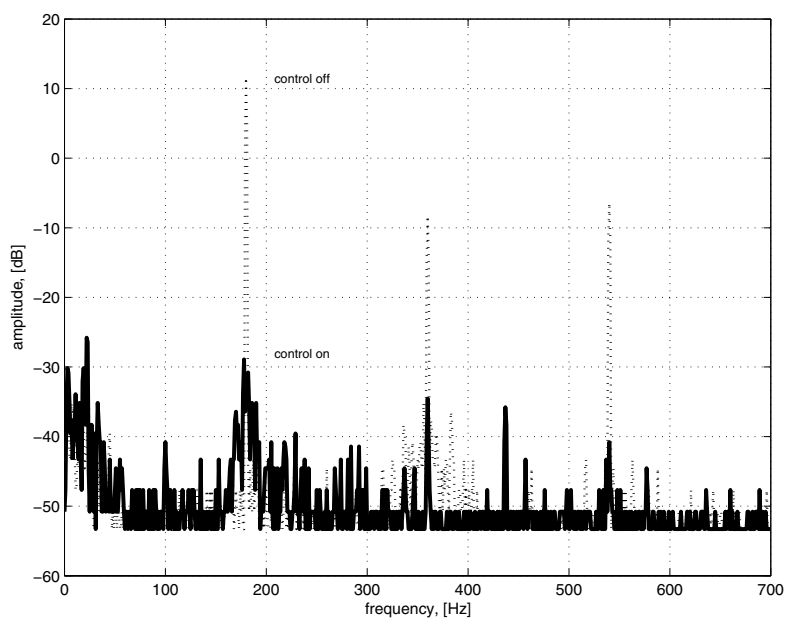

Fig. 10. Suppression of periodic noise at an error microphone. Dashed line: without control, thick solid line: with control

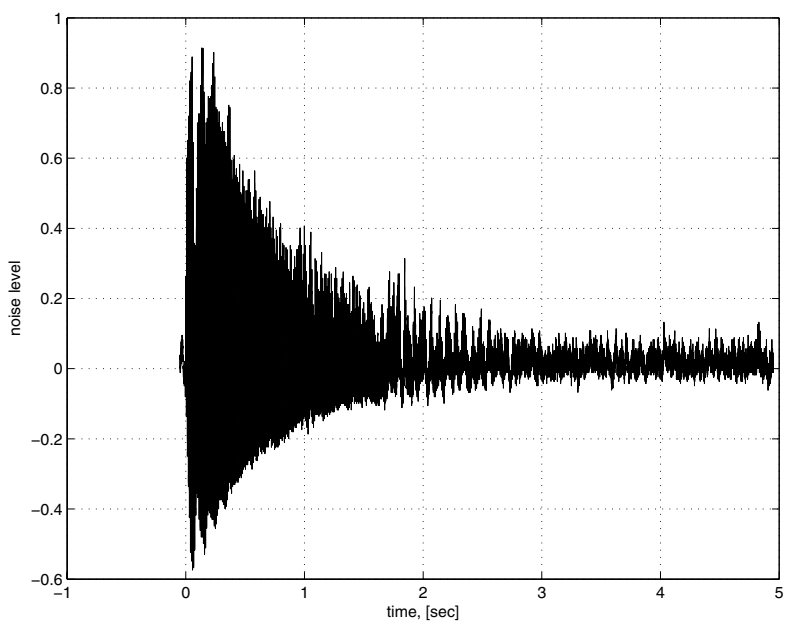

Fig. 11. Settling of the noise control system

were not carried out in an anechoic room.) Special emphasis was placed on long-time tests to observe the influence of different individual sampling frequencies or data losses. The first test results show that our system has similar performance as the appropriate wired solution.

In the following figures the suppression of a 2 by 2 system is illustrated. The motes with the microphones were placed above a chair in ear-hight, and the loudspeakers were put behind them. The signal to be suppressed was generated by another loudspeaker excited by a triangular signal of a fundamental frequency of $180 \mathrm{~Hz}$. This signal also served as reference for the controller. In Fig. 10 the spectrum of an error microphone signal can be seen. The suppression is about $40 \mathrm{~dB}$ for the component having the highest power and all the harmonics are attenuated. the settling of the system can be seen in Fig. 11. The control was turn on at time instant $0 \mathrm{sec}$, and the settling can be considered complete after $2 \ldots 3$ seconds. In order to measure the suppression in a position different from the error 


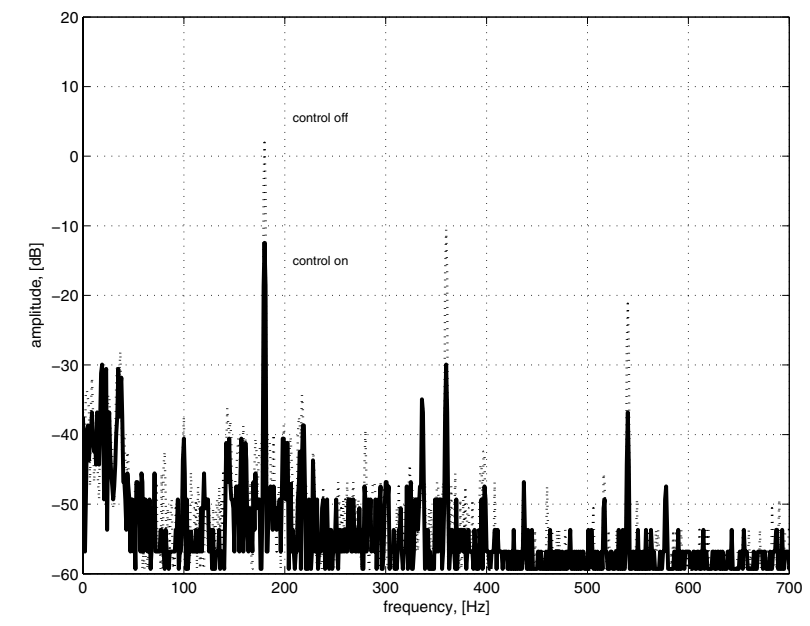

Fig. 12. Suppression of periodic noise at a test position. Dashed line: without control, thick solid line: with control

microphones, a noise level meter was used. The spectrum in Fig. 12 was calculated from the signal of the noise level meter Brüel \& Kjær 2232. The controller suppresses all the harmonics, but the suppression seems to be not complete since the components remain above the background noise level. The reason is that the signal was not captured directly at the error microphones.

The experiments have not only validated the principle of the system but show that practical restrictions (e.g. 8-bit analog to digital conversion) do not lower the performance compared to that of wired ANC. According to the first experiments the motes powered by AA batteries can operate 15-20 hours long.

\section{CONCLUSION, FUTURE WORK}

Our paper introduced a successful closed-loop wireless control application based on Berkeley MICAz motes and Analog Devices AD21061 EZ-KIT LITE board. The system realizes active noise control which is a crucial application from signal path modeling point of view. A PLL-like mechanism takes care of the synchronization, while linear interpolation fits the wireless measurement data for processing in the DSP. The noise controller is a resonator-based one which has proven its efficiency in this environment, as well. The main goal of future work is to install much more elements (i.e. microphones) in the system. This hopefully can be done by the pre-processing of samples at the motes. A further aim is the on-line identification of the secondary path.

\section{ACKNOWLEDGMENT}

This work was partly supported by the Hungarian Fund for Scientific Research under Grants OTKA TS 049743.

\section{REFERENCES}

[1] Mathiesen, M., G. Thonet, N. Aakwaag, "Wireless ad-hoc networks for industrial automation: current trends and future prospects", in: Proceedings of the IFAC World Congress, Prague, Czech Republic, July 4-8, 2005.
[2] Sujbert, L., G. Péceli, "Periodic noise cancelation using resonator based controller", in 1997 Int Symp. on Active Control of Sound and Vibration, ACTIVE '97, pp. 905-916, Budapest, Hungary, Aug. 1997.

[3] L. Sujbert, B. Vargha, "Active distortion cancelation of sinusoidal sources", IEEE Instrum. Meas. Technology Conference, May 2004, Como, Italy, in proceedings pages 322-325.

[4] G. Péceli, "A common structure for recursive discrete transforms", IEEE Trans. Circuits Syst. Vol. CAS-33 No. 10. pp. 1035-36, Oct. 1986.

[5] F. Nagy, "Measurement of signal parameters using nonlinear observers" IEEE Trans. Instrum. Meas., Vol. IM-41 No. 1. pp. 152-155, Feb. 1992.

[6] Kuo, S. M., D. R. Morgan, "Active noise control: a tutorial review", Proceedings of the IEEE Vol. 87. No. 6. pp. 943-973, June 1999.

[7] D. R. Morgan, "An analysis of multiple correlation cancelation loops with a filter in the auxiliary path" IEEE Trans. Acoust., Speech, Signal Processing vol. ASSP-28 pp. 454-467, Aug. 1980.

[8] S. J. Elliot, I. M. Stothers, P. A. Nelson, "A multiple error LMS algorithm and its application to the active control of sound and vibration", IEEE Trans. Acoust., Speech, Signal Processing vol. ASSP-35, pp. 1423-1434, Oct. 1987.

[9] Widrow, B., E. Walach, “Adaptive Inverse Control”, Prentice-Hall, Inc., 1996.

[10] Molnár, K., L. Sujbert, G. Péceli, "Synchronization of sampling in distributed signal processing systems", in Int Symp. on Intelligent Signal Processing, WISP 2003., Budapest, Hungary, Sep. 2003.

[11] www.analog.com

[12] www.xbow.com/Products

[13] www.zigbee.org 